\title{
Multiple Lung Transplant in a Patient Within 25 Years: A Case Report
}

\author{
Min Zhang, ${ }^{1}$ Ana Rita SecoAntunes, ${ }^{2}$ Romain Kessler, ${ }^{3}$ Gilbert Massard ${ }^{4}$
}

\begin{abstract}
With survival after retransplant improving over the years, issues regarding multiple retransplant have emerged. Here, we report the 25-year follow-up of a 16-year-old male patient who received 4 successful lung transplant procedures in 1990, 1991, 1995, and 2005.
\end{abstract}

Key words: Chronic graft dysfunction, Cystic fibrosis, Retransplant

\section{Introduction}

The main long-term complication after lung transplant is chronic graft dysfunction. Lung retransplant is presently the only curative strategy in the management of chronic rejection, provided that the patient appears fit for this procedure. With the growing volume of primary lung transplants, the demand for lung retransplant has also become increasingly common over the past decade. ${ }^{1-3}$ The main indications for lung retransplant include airway complications, acute graft failure, and chronic graft dysfunction. However, lung retransplant for acute graft failure and early airway complications have dramatically decreased during the past decade because of poor reported outcomes. In contrast, lung retransplant for chronic allograft dysfunction has continued as survival rates approach those obtained after first lung transplant. Several studies have shown that long-term survival rates after lung retransplant

$\overline{\text { From the }{ }^{1} \text { Department of Cardiothoracic Surgery, the First Affiliated Hospital of Chongqing }}$ Medical University, Chongqing, China; the ${ }^{2}$ Faculty of Medicine, University of Strasbourg, Strasbourg, France; and the ${ }^{3}$ Department of Respiratory Medicine and the ${ }^{4}$ Service de Chirurgie Thoracique, Hôpitaux Universitaires de Strasbourg, Strasbourg, France

Acknowledgements: The authors have no sources of funding for this study and have no conflicts of interest to declare.

Corresponding author: Gilbert Massard, Service de Chirurgie Thoracique, Hôpitaux

Universitaires de Strasbourg, 1, Place de l'hôpital, 67091 Strasbourg, France

Phone: +33607276108_E-mail: gilbert.massard@chru-strasbourg.fr

Experimental and Clinical Transplantation (2019) for chronic rejection have approached the survival rates of primary lung transplant. As survival after retransplant becomes more prolonged, complications after multiple lung retransplants emerge.

Here, we present a 25-year follow-up of a male patient with cystic fibrosis who underwent a total of 4 successive lung transplant procedures in 1990, 1991, 1995, and 2005. To our knowledge, this is one of the first cases of reported multiple lung retransplants.

\section{Case Report}

The study patient was diagnosed with cystic fibrosis at 6 years old. He was admitted to the intensive care unit with end-stage disease at age 16 years and was put on the wait list for lung transplant. After 22 days with artificial ventilation through a tracheotomy, suitable donor lungs became available and he underwent a bilateral lung transplant according to the en bloc technique with bilateral bronchial anastomoses. The procedure, performed in March 1990, showed an initially favorable clinical outcome. However, several months after transplant, lung function had deteriorated. Biopsy findings confirmed acute rejection. After failure of steroid bolus therapy, muromonabCD3 (OKT3, Johnson \& Johnson, Highland Park, NJ, USA) was administered without further success. The patient was transferred to the intensive care unit and put under artificial ventilation. Because there was an irreversible alteration of graft function, he was again placed on the emergency transplant list.

In January 1991, after 63 days of artificial ventilation, a heart-lung transplant was performed. His respiratory function gradually improved until 1993, when a pulmonary biopsy showed grade 1 rejection associated with hemosiderosis.

In September 1995, he received a left lung transplant without cardiopulmonary bypass, which resulted in a satisfactory outcome posttransplant. He was kept on the standard immunosuppressive 
regimen of the mid-1990s (combined methylprednisolone, cyclosporine, and azathioprine). In March 2004, the patient's situation again started to deteriorate. Chest computed tomography scan findings were suggestive of right-sided constrictive bronchiolitis and left-sided bronchiolitis obliterans. Bronchoalveolar lavage revealed infection with methicillin-resistant coagulase-negative Staphylococcus species. Despite treatment with vancomycin and pristinamycin, respiratory function deterioration progressed relentlessly.

In October 2005, the patient underwent right lung retransplant without extracorporeal circulation, with extubation 2 days after surgery. One month later, the patient was hospitalized for infectious bronchitis. Biology revealed coagulase-negative Staphylococcus and Enterococcus infections, which were sensitive to amoxicillin. The patient responded well to the antibiotics and was discharged.

In January 2006, the patient presented with productive cough with sputum. Microbiologic test showed relapsing Enterococcus and Staphylococcus infections. An acute transplant rejection due to superinfection was suspected, and treatment with antilymphocyte antibody (OKT3) was provided. The next 7 years were uneventful. However, in October 2013, the patient had a splenectomy for spontaneous rupture of the spleen, leading to the discovery of a posttransplant Epstein-Barr virus-related lymphoma. The latter responded well to 4 cycles of R-CHOP (cyclophosphamide, doxorubicin, vincristine, prednisone, plus rituximab). In March 2014, the patient presented with relapse of Epstein-Barr virus lymphoma. After 4 shots of rituximab, second-line chemotherapy with ifosfamide-etoposide and ofatumumab was started. Although the patient had a partial response after the first 2 cycles, performance status declined to a World Health Organization 3/4 category. The $3 \mathrm{rd}$ and 4 th cycles of treatment were poorly tolerated. After several episodes of septic shock, the patient ultimately died in July 2014.

\section{Discussion}

With survival of retransplant improving over the years, issues with regard to multiple retransplants have emerged. Lung retransplant comprises an increasing share of lung transplants, and recently patients have shown improved outcomes. The median survival of patients who undergo lung retransplant has been shown to be 2 years. ${ }^{2}$ Median survival after lung retransplant for bronchiolitis obliterans syndrome is longer than survival after retransplant for restrictive chronic lung allograft dysfunction. In a study from Thomas and colleagues, 1-year survival was only 50\% after lung retransplant for acute graft failure and airway complications; however, lung retransplant for bronchiolitis obliterans syndrome showed superior survival at 1 year $(78 \%)$ and at 5 years $(62 \%)$. These latter rates are similar to survival rates for first-time lung transplant recipients. The authors concluded that lung retransplant may be appropriate in well-selected patients with chronic lung allograft failure. ${ }^{3}$

Presently, there are no reports about outcomes of multiple lung retransplant procedures. There are many unanswered questions regarding ethics, indication, immunology, and graft tolerance, management of immunosuppression, and long-term outcomes. Our reported case demonstrates that prolonged survival is possible: this particular patient underwent 4 transplants over a 25 -year period. Two of these transplants were followed by survival of almost 10 years each.

\section{References}

1. Kawut SM. Lung retransplantation. Clin Chest Med. 2011;32(2):367377

2. Novick RJ, Stitt LW, Al-Kattan K, et al. Pulmonary retransplantation: predictors of graft function and survival in 230 patients. Pulmonary Retransplant Registry. Ann Thorac Surg. 1998;65(1):227234.

3. Thomas M, Belli EV, Rawal B, Agnew RC, Landolfo KP. Survival after lung retransplantation in the United States in the current era (2004 to 2013): better or worse? Ann Thorac Surg. 2015;100(2):452-457. 